\title{
Reconstrucción postmastectomía hilateral: estudio comparativo de satisfacción en el Servicio de Cirugía Plástica del Hospital Universitario La Paz, Madriid, Españia
}

\section{Bilateral postmastectomy reconstruction: comparative satisfaction study at the Plastic Surgery Service of La Paz University Hospital, Madrid, Spain}

\author{
Laura ÁLVAREZ MARTíNEZ*, Marta GARCÍA REDONDO** \\ Marcos ALONSO GARCÍA ${ }^{\star \star *}$
}

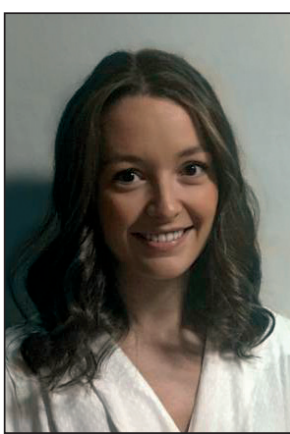

Álvarez Martínez L.
Resumen

Introducción y objetivo. La reconstrucción mamaria tras mastectomía bilateral cumple un papel fundamental para garantizar la salud psicológica y la vida social y sexual de la mujer. Las técnicas empleadas pueden ser autólogas, aloplásticas y mixtas, siendo las autólogas de elección en la mayoría de casos.

Realizamos este estudio con el fin de dilucidar si existen diferencias en cuanto a la satisfacción con el resultado final de la reconstrucción comparando las tres técnicas.

Material y método. Comparamos una serie de 35 pacientes intervenidas de reconstrucción mamaria postmastectomía bilateral, tanto inmediata como diferida en el Servicio de Cirugía Plástica del Hospital Universitario La Paz, en Madrid, España: 21 mediante técnica aloplástica, 4 con técnica autóloga y 10 con técnica mixta. Usamos 2 encuestas: una tipo Likert para valoración del resultado morfológico y funcional y una Escala Visual Analógica para valoración del resultado global. Además, valoramos la proporción de complicaciones asociadas a cada técnica y el estudio de factores de confusión sobre el resultado de la reconstrucción.

Resultados. La técnica más empleada fue la aloplástica. La técnica autóloga recibió una mejor puntuación en todas las escalas de satisfacción empleadas. Sin embargo, solo encontramos diferencias estadísticamente significativas $(\mathrm{p}=0.02)$ cuando la valoración la realizó el cirujano. En cuanto al estudio de las complicaciones, la técnica autóloga fue la que tuvo mayor proporción de complicaciones (20\%). No obstante, al comparar la aparición de complicaciones en las 3 técnicas estudiadas, únicamente encontramos diferencias estadísticamente significativas en cuanto a la aparición de necrosis cutánea $(\mathrm{p}=0.033)$ a expensas de la técnica mixta.

Conclusiones. En general, observamos una mayor satisfacción con la técnica autóloga frente al resto de técnicas, en todas las encuestas, tanto en las valoraciones del cirujano como de las pacientes, sin presentar diferencias en la proporción de complicaciones.

Palabras clave Mamoplastia, Mastectomía, Reconstrucción mamaria, Satisfacción paciente

\section{Nivel de evidencia científica Recibido [esta versión] Aceptado}

5 Significativo

22 febrero/2019

28 noviembre/2019

Conflicto de intereses: Los autores declaran no tener ningún interés financiero relacionado con el contenido de este artículo. Financiación: No hubo fuentes externas de financiación para este trabajo.

Background and objective. Breast reconstruction after bilateral mastectomy plays an essential role in guaranteeing psychological health and social and sexual life of women. The techniques used can be autologous, alloplastic or a combination of both, being autologous techniques of choice in most cases.

We conducted this study in order to elucidate whether there are differences in satisfaction regarding the final result of the reconstruction by comparing the 3 techniques.

Methods. This study compares a series of 35 patients operated on breast reconstruction after bilateral mastectomy at La Paz University Hospital in Madrid, Spain, both immediate and deferred: 21 operated on with alloplastic technique, 4 with autologous technique and 10 with mixed technique. Two surveys have been used: a Likert type survey to assess morphological and functional result and a Visual Analogue Scale for the assessment of the overall result. In addition, we reviewed the proportion of complications associated with each technique and the study of confounding factors on the outcome of the reconstruction.

Results. The most commonly used technique in this series was the aloplastic one. The autologous technique was the one that received the best score in all the satisfaction scales used. However, statistically significant differences are only found when the evaluation is performed by the surgeon $(p=0.02)$. Regarding the study of complications, the autologous technique had the highest proportion of complications (20\%). Nevertheless, when comparing the appearance of complications in the 3 techniques, only statistically significant differences were found in the appearance of skin necrosis $(p=0.033)$, at the expense of the mixed technique.

Conclusions. In general, greater satisfaction was observed with the autologous technique compared to the rest of them, in all the surveys, both in the surgeon's assessments and those of the patients, with no differences in the proportion of complications.

Key words Mammoplasty, Mastectomy, Patient satisfaction

\section{Level of evidence Received [this version]}

5 Meaningfulness

22 February/2019

28 November $/ 2019$ 


\section{Introducción}

La mama es un órgano glandular que además de su función de producción de leche, desempeña un importante papel en la figura del cuerpo de la mujer así como en el desarrollo de su vida social y sexual. Por este motivo y dado que la mastectomía es una parte fundamental en el tratamiento del cáncer de mama, tan prevalente hoy en día, la reconstrucción de este órgano cobra gran importancia en la actualidad. Asimismo, y dado el gran impacto psicológico que ello supone para la mujer, en este proceso reconstructivo es clave evaluar el grado de satisfacción que las pacientes obtienen con las distintas técnicas quirúrgicas. ${ }^{(1)}$

La reconstrucción mamaria postmastectomía puede realizarse de forma inmediata (en la misma cirugía que la extirpación de la mama) o diferida (al cabo de meses o años). La técnica reconstructiva a realizar ha de ser elegida teniendo en cuenta las características morfológicas y personales de cada paciente, así como los tratamientos adyuvantes a los que ha sido o va a ser sometida.

La reconstrucción mamaria autóloga consiste en el uso de tejido de la propia paciente para la creación de la nueva mama. Las complicaciones más importantes en esta técnica son: hematoma, linfocele, necrosis cutánea, necrosis del colgajo, hernias de zona donante y dehiscencias. ${ }^{(2)}$ La técnica aloplástica, consiste en la utilización de un implante para reproducir la forma de la mama. Se puede usar una prótesis mamaria, un expansor mamario o un dispositivo mixto (expansor-prótesis). Las complicaciones más frecuentemente asociadas a esta técnica son: hematoma, infección, extrusión protésica, necrosis cutánea, contractura capsular y rotura protésica. ${ }^{(3)} \mathrm{La}$ reconstrucción con técnica mixta consiste en el uso de un colgajo pediculado autólogo (parte autóloga) combinado con un implante, ya sea una prótesis, un expansor o un expansor-prótesis (parte aloplástica). Esta técnica puede presentar las complicaciones tanto de la técnica autóloga como de la aloplástica. ${ }^{(2)}$

A pesar de que cada técnica tiene sus ventajas e inconvenientes, en nuestra opinión la reconstrucción autóloga es la técnica de referencia en la mayoría de los casos, ya que con ella se pueden conseguir resultados óptimos en cuanto a textura, volumen y simetría, evitando el uso de implantes mamarios con las complicaciones que conllevan. Además, es la técnica más estable a lo largo de la vida de la paciente. ${ }^{(4-6)}$

En esta línea, hemos realizado un estudio comparativo de la satisfacción de de las pacientes sometidas a reconstrucción mamaria con las distintas técnicas empleadas por el Servicio de Cirugía Plástica del Hospital Universitario La Paz, en Madrid, España. El objetivo de este trabajo es comparar el resultado en cuestión de satisfacción global, evaluada por las pacientes y un cirujano, entre las distintas técnicas quirúrgicas reconstructivas (autólogas, aloplásticas y mixtas) en pacientes intervenidas de mastectomía bilateral, teniendo en cuenta tanto las reconstrucciones inmediatas como diferidas.

\section{Material y método}

Realizamos un estudio observacional, analítico, longitudinal retrospectivo sobre una población de estudio constituida por mujeres a las que se les practicó reconstrucción mamaria después de mastectomía bilateral, tanto inmediata como diferida. Seleccionamos mujeres intervenidas por este motivo durante los años 2015-2017 en el Servicio de Cirugía Plástica y Reconstructiva del Hospital Universitario La Paz (HULP) en Madrid (España). Inicialmente recolectamos los datos de 90 pacientes, de las cuales 44 se negaron a participar en el estudio o fue imposible contactar con ellas. Citamos a las 46 pacientes restantes, de las cuales 11 no acudieron a la cita, por lo que la muestra final fue de 35 pacientes.

Dado que el objetivo principal del trabajo consiste en valorar si existen diferencias estadísticamente significativas en cuanto a satisfacción global entre las diferentes técnicas reconstructivas, utilizamos como variables principales: satisfacción global, valorada con Escala Visual Analógica (EVA) por el cirujano y por las pacientes; y satisfacción morfológica y funcional, evaluadas con escala Likert.

La escala EVA, instrumento validado diseñado por el EuroQol-5D, ${ }^{(7)}$ valora la satisfacción a través de una línea milimetrada de $20 \mathrm{~cm}$ que comprende valores desde 0 , peor estado de salud imaginable; en nuestro caso adaptado a peor grado de satisfacción con la reconstrucción mamaria, hasta 100, mejor estado de salud imaginable; adaptado en nuestro caso a mejor grado de satisfacción con la reconstrucción mamaria.

Para la valoración de la satisfacción de los aspectos morfológicos y funcionales de la reconstrucción elaboramos una encuesta tipo Likert basada en el contenido de los cuestionarios validados QLQ-C30 ${ }^{(8)}$ y QLQ-BR23, ${ }^{(9)}$ cuestionarios de la European Organisation for Research and Treatment of Cancer. La encuesta valora del 1, muy mal/muy en desacuerdo, al 5, muy bien/muy de acuerdo, aspectos morfológicos evaluados por cirujano y paciente: color, textura, cicatriz, simetría, tamaño, naturalidad, complejo areola-pezón y satisfacción global del resultado morfológico; y aspectos funcionales, valorados sólo por las pacientes: cumplimiento de las expectativas, grado de satisfacción con la imagen corporal vestida y desnuda, influencia sobre la vida sexual, satisfacción con 


\section{Tabla I. Encuestas de satisfacción morfológica y funcional}

\author{
Encuesta de Evaluación Morfológica
}

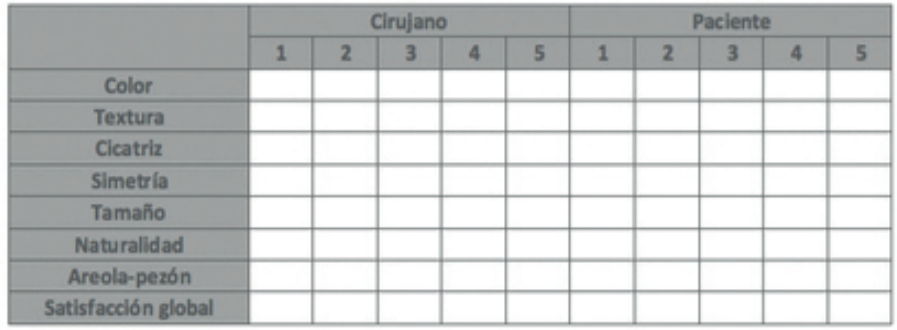

1. Nada contenta

2. Poco contenta

3. Ni contenta ni descontenta

4. Contenta

5. Muy contenta

\section{Encuesta de Evaluación Funcional}

A. ¿La reconstrucción ha respondido a sus expectativas?

$\begin{array}{ccccc}1 & 2 & 3 & 4 & 5 \\ \text { Nada } & \text { Poco } & \text { Regular } & \text { Bastante } & \text { Mucho }\end{array}$

B. ¿Estra contenta con el resultado de la reconstrucción vestida?

$\begin{array}{ccccc}1 & 2 & 3 & 4 & 5 \\ \text { Nada } & \text { Poco } & \text { Regular } & \text { Bastante } & \text { Mucho }\end{array}$

C. ¿Está contenta con el resultado de la reconstrucción desnuda?

$\begin{array}{ccccc}1 & 2 & 3 & 4 & 5 \\ \text { Nada } & \text { Poco } & \text { Regular } & \text { Bastante } & \text { Mucho }\end{array}$

D. ¿Ha influido la reconstrucción mamaria en su vida sexual?

$\begin{array}{ccccc}1 & 2 & 3 & 4 & 5 \\ \text { Nada } & \text { Poco } & \text { Regular } & \text { Bastante } & \text { Mucho }\end{array}$

E. ¿Está contenta con el grado de sensibilidad residual?

$\begin{array}{ccccc}1 & 2 & 3 & 4 & 5 \\ \text { Nada } & \text { Poco } & \text { Regular } & \text { Bastante } & \text { Mucho }\end{array}$

F. En función de su resultado, ċrecomendaría este tipo de reconstrucción mamaria?

$\begin{array}{ccccc}1 & 2 & 3 & 4 & 5 \\ \text { Nada } & \text { Poco } & \text { Regular } & \text { Bastante } & \text { Mucho }\end{array}$

la sensibilidad residual, y si recomendaría la técnica reconstructiva a la que haya sido sometida a otras pacientes. Mostramos ambas encuestas en la Tabla I.

Además, recogemos otras variables como: edad de la paciente; factores de riesgo: hábito tabáquico y factores de riesgo cardiovascular; y variables relacionadas con la patología: exposición a radioterapia previa a la reconstrucción, administración de quimioterapia (quimioterapia y hormonoterapia) y complicaciones postquirúrgicas (hematoma, dehiscencia, cicatriz patológica, seroma, necrosis del colgajo, contractura capsular, rotura protésica, y extrusión protésica).

El proceso de recogida de datos y selección de pacientes lo realizamos a través de la memoria de quirófano del Servicio de Cirugía Plástica y Reconstructiva del HULP. Una vez recogidos los datos de las pacientes que cumplían los criterios de inclusión procedimos a llamarlas telefónicamente, informándoles de los detalles del estudio para citar a aquellas que aceptasen participar en el mismo. A las pacientes que acudieron a la cita en las consultas del HULP se les informó de las características y procedimiento del estudio, verbalmente y por escrito, mediante un consentimiento informado que debían leer y firmar previamente a la realización de las encuestas.

Los datos recogidos fueron analizados estadísticamente utilizando el programa SPSS versión 24. Llevamos a cabo un análisis descriptivo de los resultados reflejados en cuanto a satisfacción por la paciente y por el cirujano y valoramos si existían diferencias en los resultados en función de la técnica empleada mediante el test de Kruskal-Wallis y un análisis individual cuando encontramos diferencias. Por otro lado, hicimos un análisis descriptivo de las complicaciones con cada técnica y un análisis de regresión lineal múltiple para la variable satisfacción global. Además, analizamos qué aspecto de la valoración morfológica y funcional es el peor y el mejor valorado para cada técnica. Para ello consideramos como aspectos mejor valorados aquellos que reúnen un mayor porcentaje de opiniones "buenas" y "muy buenas". Del mismo modo, lo peor valorado es aquello que obtenga una mayor proporción de puntuaciones "malas" y "muy malas". Por último, estudiamos mediante pruebas de regresión lineal, si alguna de las siguientes variables se comportó como un factor de confusión para el resultado de satisfacción global conjunta; para ello calculamos la media, en cada caso, de paciente y EVA cirujano, sin estratificar la muestra por técnica reconstructiva: edad, tabaquismo, factores de riesgo cardiovascular, administración de quimioterapia y administración de radioterapia.

El estudio tuvo la autorización por parte del Director del Departamento de Cirugía de la Universidad Autónoma de Madrid (UAM) y fue aprobado por el Comité de Ética de Investigación del HULP, así como por el Subcomité de Ética de la Facultad de Medicina de la UAM. 


\section{Resultados}

De todas las pacientes recogidas en nuestro grupo de estudio, 21 fueron reconstruidas mediante técnica aloplástica (60\%), 4 mediante técnica autóloga $(11.4 \%)$ y 10 mediante técnica mixta $(28.6 \%)$. Del total de pacientes, 12 (10 pacientes intervenidas mediante técnica mixta y 2 pacientes del grupo tratado con técnica aloplástica) todavía no habían completado la reconstrucción del complejo areola-pezón, a la espera de realizarse el tatuaje del complejo. La edad media de la muestra fue de 49.7 años con un IC del 95\% (46.4-52.9 años), siendo la edad mínima 32 años y la máxima 69 años. Un $40 \%$ de las mujeres de la muestra nunca habían fumado, mientras que un $60 \%$ de ellas sí había estado expuesta a los efectos del tabaco (48.6\% eran exfumadoras y $11.4 \%$ fumadoras activas). El $8.6 \%$ de las pacientes eran hipertensas y el $20 \%$ padecían dislipemia. El $2.9 \%$ de las mujeres participantes tenían diabetes mellitus. El índice de masa corporal (IMC) medio de la muestra fue de 25.4 con un IC del 95\% (23.9-26.8). Un 54.3\% de las pacientes recibieron tratamiento radioterápico previo a la reconstrucción; y un $80 \%$ de las mujeres recibieron quimioterapia. En la Tabla II describimos las características de la muestra de manera global y en función de la técnica quirúrgica empleada para la reconstrucción mamaria.

El resultado de la cirugía fue valorado por parte del cirujano mediante 2 encuestas: una encuesta de evaluación morfológica y escala EVA. Por otro lado, las pacientes valoraron el resultado mediante: encuesta de evaluación morfológica, encuesta de evaluación funcional (la evaluación funcional se analiza de forma conjunta con la evaluación morfológica, mediante el sumatorio de la puntuación en cada ítem, siendo la máxima puntuación 65 y la mínima 13) y EVA.

Los resultados obtenidos (Tabla III), muestran que la técnica autóloga es la que recibió mejores puntuaciones en todas las escalas, seguida de la técnica aloplástica y por último, de la técnica mixta. Sobre un total de 100, las puntuaciones obtenidas en cada técnica fueron las siguientes. En la técnica autóloga: escala EVA cirujano 77.5; evaluación morfológica del cirujano 87.14; escala

\section{Tabla II. Características de la muestra}

\begin{tabular}{|c|c|c|c|c|c|c|c|c|c|}
\hline & & \multicolumn{2}{|c|}{$\begin{array}{c}\text { TOTAL } \\
(n-35)\end{array}$} & \multicolumn{2}{|c|}{$\underset{(n=4)}{\text { AUTÓLOGA }}$} & \multicolumn{2}{|c|}{$\underset{(n-21)}{\operatorname{ALOPL} \text { ĹSTICA }}$} & \multicolumn{2}{|c|}{$\underset{(n-10)}{\operatorname{MixTA}}$} \\
\hline & & $\mathbf{N}$ & $\%$ & $\mathbf{N}$ & $\%$ & $\mathbf{N}$ & $\%$ & $\mathbf{N}$ & $\%$ \\
\hline \multirow{4}{*}{$\begin{array}{l}\text { EDAD } \\
\text { (años) }\end{array}$} & $30-39$ & 5 & 14,3 & 1 & 25 & 2 & 9,5 & 2 & 20 \\
\hline & $40-49$ & 12 & 34,3 & 1 & 25 & 9 & 42,9 & 2 & 20 \\
\hline & $50-59$ & 13 & 37,1 & 2 & 50 & 5 & 23,8 & 6 & 60 \\
\hline & $60-69$ & 5 & 14,3 & 0 & 0 & 5 & 23,8 & 0 & 0 \\
\hline \multirow{3}{*}{ IMC $^{*}$} & $\begin{array}{c}\text { Normal } \\
\text { (IMC=18,5-24,99) }\end{array}$ & 14 & 40 & 1 & 25 & 10 & 47,6 & 3 & 30 \\
\hline & $\begin{array}{l}\text { Sobrepeso } \\
\text { (IMC-25-25,99) }\end{array}$ & 18 & 51,4 & 3 & 75 & 10 & 47,6 & 5 & 50 \\
\hline & $\begin{array}{c}\text { Obesidad } \\
\text { (IMC } \supseteq 30)\end{array}$ & 3 & 8,6 & 0 & 0 & 1 & 4,8 & 2 & 20 \\
\hline \multirow{3}{*}{ TABAQUISMO } & No fumadoras & 14 & 40 & 4 & 100 & 9 & 42,9 & 1 & 10 \\
\hline & Exfumadoras & 17 & 48,6 & 0 & 0 & 10 & 47,6 & 7 & 70 \\
\hline & $\begin{array}{c}\text { Fumadoras } \\
\text { activas }\end{array}$ & 4 & 11,4 & 0 & 0 & 2 & 9,5 & 2 & 20 \\
\hline \multirow{2}{*}{ HTA $^{*}$} & $\mathbf{S i}$ & 3 & 8,6 & 1 & 25 & 0 & 0 & 2 & 20 \\
\hline & No & 32 & 91,4 & 3 & 75 & 21 & 100 & 8 & 80 \\
\hline \multirow{2}{*}{$\mathbf{D M}^{*}$} & $\mathbf{S i}$ & 1 & 2,9 & 0 & 0 & 0 & 0 & 1 & 10 \\
\hline & No & 34 & 97,1 & 4 & 100 & 21 & 100 & 9 & 90 \\
\hline \multirow{2}{*}{$\mathrm{DL}^{*}$} & $\mathbf{S i}$ & 7 & 20 & 2 & 50 & 4 & 19 & 1 & 10 \\
\hline & No & 28 & 80 & 2 & 50 & 17 & 81 & 9 & 90 \\
\hline \multirow{2}{*}{$\mathbf{R T}^{*}$} & $\mathbf{S i}$ & 19 & 54,3 & 3 & 75 & 8 & 38,1 & 8 & 80 \\
\hline & No & 16 & 45,7 & 1 & 25 & 13 & 61,9 & 2 & 20 \\
\hline \multirow{2}{*}{$\mathbf{Q T}{ }^{*}$} & $\mathbf{S i}$ & 28 & 80 & 4 & 100 & 16 & 76,2 & 8 & 80 \\
\hline & No & 7 & 20 & 0 & 0 & 5 & 23,8 & 2 & 20 \\
\hline
\end{tabular}

*IMC: Índice de Masa Corporal; HTA: Hipertensión Arterial; DM: Diabetes Mellitus; DL: Dislipemia; RT: Radioterapia; QT: Quimioterapia 


\section{Tabla III. Análisis estadístico descriptivo de las encuestas de satisfacción}

\begin{tabular}{|c|c|c|c|c|c|c|c|}
\hline & & \multicolumn{2}{|c|}{$\begin{array}{c}\text { Puntuaciones } \\
\text { cirujano }\end{array}$} & \multicolumn{4}{|c|}{$\begin{array}{l}\text { Puntuaciones } \\
\text { paciente }\end{array}$} \\
\hline & & $\begin{array}{c}\text { Escala } \\
\text { EVA }\end{array}$ & $\begin{array}{c}\text { Evaluación } \\
\text { morfológica }\end{array}$ & $\begin{array}{c}\text { Escala } \\
\text { EVA }\end{array}$ & $\begin{array}{c}\text { Evaluación } \\
\text { morfológica }\end{array}$ & $\begin{array}{c}\text { Evaluación } \\
\text { funcional }\end{array}$ & $\begin{array}{c}\text { Evaluación } \\
\text { global }\end{array}$ \\
\hline GENERAL & $\begin{array}{c}\text { Mediana } \\
\text { (Rango) }\end{array}$ & $\begin{array}{c}70.0 \\
(70.0)\end{array}$ & $\begin{array}{l}82.86 \\
(57.14)\end{array}$ & $\begin{array}{l}80.0 \\
(85.0)\end{array}$ & $\begin{array}{c}80.0 \\
(65.71)\end{array}$ & $\begin{array}{c}80.0 \\
(66.67)\end{array}$ & $\begin{array}{l}76.92 \\
(52.31)\end{array}$ \\
\hline ALOPLÁSTICA & $\begin{array}{c}\text { Mediana } \\
\text { (Rango) }\end{array}$ & $\begin{array}{l}70.0 \\
(70.0)\end{array}$ & $\begin{array}{l}82.86 \\
(57.14)\end{array}$ & $\begin{array}{l}80.0 \\
(70.0)\end{array}$ & $\begin{array}{c}80.0 \\
(57.14)\end{array}$ & $\begin{array}{c}80.0 \\
(63.33)\end{array}$ & $\begin{array}{l}78.46 \\
(52.31)\end{array}$ \\
\hline AUTÓLOGA & $\begin{array}{c}\text { Mediana } \\
\text { (Rango) }\end{array}$ & $\begin{array}{l}77.5 \\
(20.0)\end{array}$ & $\begin{array}{l}87.14 \\
(11.43)\end{array}$ & $\begin{array}{l}90.0 \\
(25.0)\end{array}$ & $\begin{array}{l}88.57 \\
(8.57)\end{array}$ & $\begin{array}{l}86.67 \\
(23.33)\end{array}$ & $\begin{array}{l}89.23 \\
(12.31)\end{array}$ \\
\hline MIXTA & $\begin{array}{c}\text { Mediana } \\
\text { (Rango) }\end{array}$ & $\begin{array}{c}57.5 \\
(40.0)\end{array}$ & $\begin{array}{l}65.71 \\
(42.86)\end{array}$ & $\begin{array}{l}72.5 \\
(85.0)\end{array}$ & $\begin{array}{l}65.71 \\
(60.0)\end{array}$ & $\begin{array}{l}75.00 \\
(46.67)\end{array}$ & $\begin{array}{l}73.08 \\
(43.08)\end{array}$ \\
\hline
\end{tabular}

EVA paciente 90; evaluación morfológica paciente 88.57; evaluación funcional paciente 86.67 ; evaluación global de la paciente 89.23. En la técnica aloplástica: EVA cirujano 70; evaluación morfológica cirujano 82.86; EVA paciente, evaluación morfológica y funcional de paciente 80 ; evaluación global de la paciente 78.46 . Por último, en la técnica mixta: EVA cirujano 57.5; evaluación morfológica cirujano 65.71; EVA paciente 72.5 ; evaluación morfológica paciente 65.71; evaluación funcional paciente 75; y evaluación global de la paciente 73.08.

Cuando comparamos la puntuación EVA de la paciente en función de la técnica, no encontramos diferencias estadísticamente significativas $(\mathrm{p}=0.16)$. Sin embargo, sí se muestran diferencias estadísticamente significativas entre las técnicas según la valoración EVA del cirujano $(\mathrm{p}=0.02)$.

Debido al resultado no significativo con la EVA medido por la paciente, realizamos el mismo procedimiento comparando las 3 técnicas en función de la puntuación morfológica global de la paciente y del cirujano con el fin de evaluar los aspectos más objetivos de la encuesta. Los resultados obtenidos muestran que no hay diferencias estadísticamente significativas en la puntuación morfológica de la paciente $(\mathrm{p}=0.08)$, pero sí se encontraron cuando esta comparación fue realizada en función de la puntuación morfológica del cirujano $(\mathrm{p}=0.02)$.

Estudiamos también las complicaciones más representativas dependientes de cada técnica. Un $6.12 \%$ de las pacientes intervenidas mediante técnica aloplástica sufrieron complicaciones, siendo la más frecuente la contractura capsular periprotésica, que supuso un $2.7 \%$ del total de las pacientes intervenidas con esta técnica, lo que corresponde a un $45 \%$ de todas las complicaciones (Gráfico 1).
Un 20\% de las pacientes intervenidas con técnica autóloga sufrieron complicaciones, siendo la más frecuente la dehiscencia que supuso un $10 \%$ del total de pacientes en este grupo y un $50 \%$ de las complicaciones totales (Gráfico 2). Un $17.5 \%$ de pacientes intervenidas con técnica mixta sufrieron complicaciones, siendo la más frecuente la necrosis del colgajo y la extrusión protésica que supusieron cada una un $3.8 \%$ del total de las pacientes intervenidas mediante esta técnica y un $22 \%$ del total de complicaciones (Gráfico 3).

Al comparar las 3 técnicas, obtuvimos diferencias estadísticamente significativas en cuanto a la aparición de necrosis $(\mathrm{p}=0.033)$ a expensas de la técnica mixta.

Mostramos los resultados obtenidos de la encuesta morfológica evaluada por el cirujano en el Gráfico 4, destacando el $100 \%$ de valoraciones positivas (es decir, valoradas por las pacientes como "bien" o "muy bien") en el caso del color en la técnica aloplástica, y el tamaño, naturalidad y complejo areola-pezón en la técnica autóloga; y un $80 \%$ de valoraciones positivas para el tamaño en la técnica mixta. En cuanto a las valoraciones negativas (aquellas valoradas como "mal" o "muy mal"), la simetría fue el aspecto peor valorado, con un $23.8 \%$ de valoraciones negativas en la técnica aloplástica y un $40 \%$ en la técnica mixta) más otro $40 \%$ que creemos importante señalar de valoración como regular en esta técnica. Por otro lado, la naturalidad también supuso un $40 \%$ de valoraciones negativas en la técnica mixta.

Cabe destacar los resultados de valoración del complejo areola-pezón en el grupo de pacientes intervenidas con técnica mixta, en el cual hay un $100 \%$ de valoraciones "regular", debido a que por el escaso tiempo de evolución desde la cirugía, ninguna de ellas se había realizado el tatuaje del complejo. 

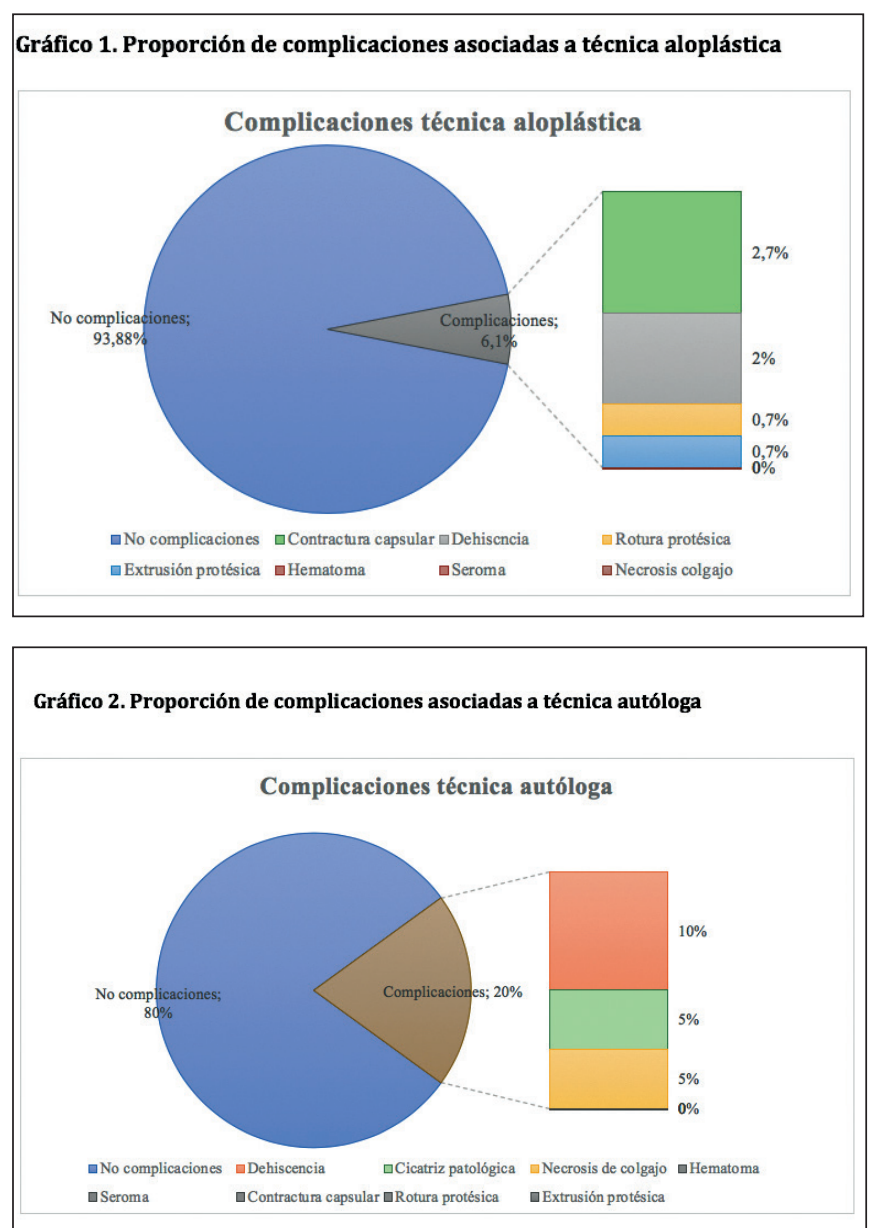

Gráfico 3. Proporción de complicaciones asociadas a técnica mixta

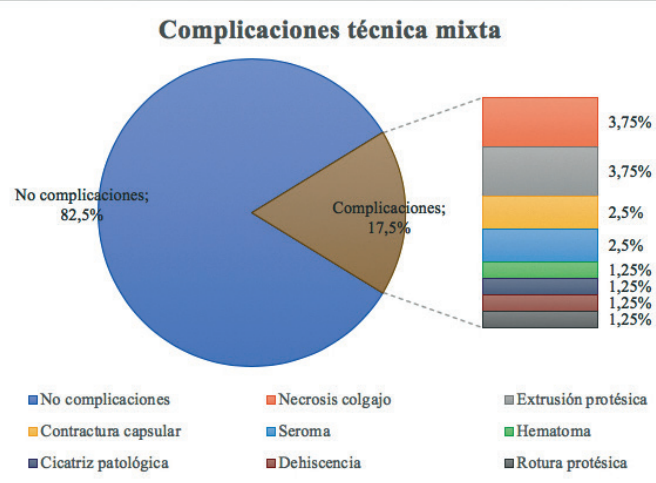

Ilustramos los datos recogidos en cuanto a la valoración morfológica por parte de la paciente en el Gráfico 5 , destacando el $100 \%$ de valoraciones positivas (valoraciones "bien" y "muy bien") en el caso del color en la técnica aloplástica. Además, todos los parámetros tienen un $100 \%$ de valoraciones positivas en el caso de la técnica autóloga, excepto la valoración del complejo areola-pezón que tiene un $50 \%$ de valoraciones positivas y un $50 \%$ de valoraciones "regular".

En la parte negativa (valoraciones "mal" y "muy mal"), la simetría ( $40 \%$ valoraciones negativas en técnica mixta y $23.8 \%$ en el caso de la técnica aloplástica) y el complejo areola-pezón ( $23.8 \%$ de valoraciones negativas en técnica aloplástica, $50 \%$ de valoraciones "regular" en técnica autóloga y 100\% de valoraciones "regular" en técnica mixta) son los aspectos peor valorados. Al igual que ocurre con la evaluación del cirujano, el complejo areola-pezón está valorado en todos los casos como "regular" debido a la reconstrucción incompleta del mismo.

Respecto a la evaluación funcional referida por la paciente resumimos los datos en el Gráfico 6, destacando el cumplimiento de las expectativas, la figura vestida y el grado de recomendación de la técnica a otras posibles pacientes, con el $100 \%$ de valoraciones positivas en el caso de la técnica autóloga; así como un $95.2 \%$ de valoraciones positivas para la figura vestida y un $90.5 \%$ de grado de recomendación en técnica aloplástica; y un $90 \%$ de valoraciones positivas para el grado de recomendación en el caso de la técnica mixta.

En la parte negativa, destaca la técnica mixta con un $40 \%$ de valoraciones negativas en las consecuencias sobre la vida sexual, un $30 \%$ de valoraciones negativas en cuanto a la figura desnuda y un $30 \%$ de valoraciones negativas y un 50\% de valoraciones "regular" para la sensibilidad residual.

En el análisis multivariante de regresión lineal múltiple, controlando posibles factores de confusión (edad, IMC, tabaquismo, hipertensión arterial, dislipemia, diabetes mellitus, quimioterapia y radioterapia) respecto a la variable "EVA media", la única relación resultante con significación estadística fue la exposición al tabaco $(\mathrm{p}=0.018)$ (coeficiente de regresión -0.447).

\section{Discusión}

Este estudio fue realizado con una serie de pacientes tratadas quirúrgicamente por el Servicio de Cirugía Plástica del Hospital Universitario La Paz de Madrid, España. Se trata de un hospital público de tercer nivel, vinculado a la Universidad Autónoma de Madrid, que atiende sanitariamente a los pacientes del área Norte de Madrid y a otros pacientes de distintas localidades vecinas por ser un centro reconocido de referencia y excelencia. Este Servicio de Cirugía Plástica, consta de una Unidad de Patología Mamaria, equipo multidisciplinar formado por distintos especialistas: Ginecología, Medicina Nuclear, Cirugía General, Cirugía Plástica y Reparadora, Oncología Médica, Oncología Radioterápica, Anatomía Patológica y Psiquiatría, además de personal de enfermería especializado. La unidad atiende más de 250 nuevos casos al año, habiendo realizado más de 6000 reconstrucciones mamarias desde su creación hace aproximadamente 40 años. ${ }^{(10)}$

Un aspecto interesante a tener en cuenta en nuestro estudio es que el grupo que consta de un mayor número de pacientes es el tratado mediante técnica aloplástica, a 
pesar de que en nuestra opinión, la técnica de referencia para la reconstrucción mamaria bilateral es la autóloga. Bajo nuestro punto de vista, esta diferencia se debe en el Servicio de Cirugía Plástica del HULP y teniendo en cuenta el periodo temporal de selección de la muestra, a la optimización de recursos adaptada al Sistema Nacional de Salud Público en España, del que depende nuestro centro, que responde a criterios de eficiencia sanitaria.

En cuanto al análisis del estudio objeto de esta publicación, hemos obtenido resultados dispares en función de si analizamos los mismos con la puntuación dada por el cirujano o por la paciente. La discrepancia entre los resultados, en función del evaluador, pueden deberse a distintos motivos. El primero a considerar es el limitado tamaño muestral presente en este estudio. Por otro lado, las diferencias podrían deberse a un sesgo de obsequiosidad, es decir, a que las pacientes hayan hecho sus valoraciones con el objetivo de contentar al cirujano, dado que hemos encontrado mejores puntuaciones por parte de las pacientes al analizar la puntuación según la escala; o a un sesgo de subjetividad, debido a la vivencia personal del proceso de cada paciente. Existen trabajos en la literatura $^{(11,12)}$ en los que se evalúa la satisfacción y la calidad de vida en pacientes reconstruidas con técnica autóloga y aloplástica que describen, al igual que en nuestro caso, que las mujeres reconstruidas mediante técnica autóloga están más satisfechas que las pacientes reconstruidas con técnica aloplástica. Sin embargo, a diferencia de estos estudios, en el nuestro no encontramos diferencias estadísticamente significativas entre ambas técnicas si bien vimos mejores puntuaciones con la técnica autóloga. Esta no coincidencia de resultados puede tener múltiples motivos, entre los que destacamos el tamaño muestral, una escala de evaluación distinta y unos criterios de inclusión más estrictos que los nuestros. No encontramos resultados en la literatura para estudios que comparen dichas técnicas con la técnica reconstructiva mixta.

Las complicaciones han sido valoradas en múltiples investigaciones ${ }^{(13-17)}$. En el estudio de Varela y col. ${ }^{(13)}$ la complicación más frecuente asociada a la reconstrucción mamaria autóloga (con colgajo DIEP) es la necrosis grasa $(8.2 \%)$, seguida del hematoma (3\%); a diferencia de nuestro estudio, donde fue la dehiscencia (10\%). En cuanto a la técnica aloplástica, la mayoría de estudios analizan las complicaciones en función de si la reconstrucción es inmediata o diferida, lo cual no se tuvo en cuenta en nuestro estudio. En ellos, la complicación más frecuentemente asociada es la contractura capsular, ${ }^{(14-17)}$ al igual que en nuestro caso. No encontramos información con la que comparar nuestros resultados en cuanto a la técnica mixta.

En nuestro estudio, el único factor de riesgo estadísticamente significativo tras el análisis multivariante fue el tabaquismo, lo que contrasta con otros estudios donde los factores que resultan significativos son el IMC, ${ }^{(18)}$ la presencia de radioterapia previa ${ }^{(19)}$ o la presencia de obesidad, tabaquismo e hipertensión arterial. ${ }^{(20)}$ A pesar de los resultados obtenidos, es sabido que los factores estudiados (edad, hipertensión arterial, diabetes mellitus, dislipemia, obesidad, quimioterapia y radioterapia) conllevan una peor capacidad cicatricial y regenerativa dado que se asocian a una mala salud vascular, que dificulta que los procesos de regeneración tisular se desarrollen de una manera adecuada. ${ }^{(21)}$

Nuestro estudio presenta una serie de limitaciones, como puede ser un periodo de recogida de datos muy cercano a la actualidad, lo que supone que una parte importante de las pacientes no hayan finalizado la reconstrucción (por falta de reconstrucción del complejo areola-pezón), lo cual ha podido infravalorar el grado de satisfacción con el resultado. También que el tamaño muestral final es pequeño, influido principalmente por la necesidad de realizar la encuesta de forma presencial lo que ha supuesto un gran número de pérdidas de pacientes; no obstante, este punto se podría solucionar si hubiésemos aumentado el marco temporal en el que se realizaron las reconstrucciones. Las encuestas morfológica y funcional usadas en el trabajo no son cuestionarios validados; sin embargo las hemos utilizado por incluir aspectos fundamentales en dichas valoraciones tales como el complejo areola-pezón, que no incluyen los modelos actuales del QLQ-BR23 y QLQ-C30, y dado que por otro lado, todavía se encuentra en fase III la versión específica de reconstrucción mamaria QLQ-BRECON23, ${ }^{(22)}$ que sería la ideal para este estudio.

\section{Conclusiones}

En conclusión, entre las pacientes de nuestro grupo de estudio hemos observado mejores resultados en todas las encuestas de satisfacción con la técnica autóloga y no ha habido diferencias significativas en cuanto a la aparición de complicaciones en función de la técnica reconstructiva empleada.

\section{Dirección del autor}

Dra. Laura Álvarez Martínez

Servicio de Cirugía Plástica

Hospital Universitario La Paz

Paseo de la Castellana 261

28046 Madrid, España

Correo electrónico: 1.alvarez.martinez94@gmail.com 


\section{Bibliografía}

1. Parker PA. Breast reconstruction and psychosocial adjustment: what have we learned and where do we go from here? Seminars Plast Surg 2004;18: 131-138.

2. Fitoussi A. Oncoplastic and reconstructive surgery for breast cancer. Paris, France: Springer, 2014; Pp.59-88.

3. Fitoussi A. Oncoplastic and reconstructive surgery for breast cancer. Paris, France: Springer, 2014; Pp.43-54.

4. Cabrera Sánchez, E. et al. Satisfacción en pacientes con reconstrucción mamaria con colgajo D.I.E.P. Cir. plást. iberolatinoam., 2006, 32(3): 169-179.

5. Fee-Fulkerson, K., Conaway, M.R., Winer, E.P. Factors Contributing to Patient Satisfaction with Breast Reconstruction Using Silicone Gel Implants. Plast Reconst. Surg, 1996; 97(4): 1420 .

6. Zhong T, McCarthy CM, Price AN, Pusic AL. Evidencebased medicine: breast reconstruction. Plast Reconst Surg, 2013;132(6):1658-1669.

7. Herdman M, Badia X, Berra S. El EuroQol-5D: una alternativa sencilla para la medición de la calidad de vida relacionada con la salud en atención primaria. Aten Primaria 2001; 28(6): 425-430.

8. EORTC - Quality of Life (2018). Quality of Life of Cancer Patients [online] Available at: http://groups.eortc.be/qol/eortcqlq-c30 [Accessed 1 May 2018].

9. EORTC - Quality of Life (2018) Module for Breast Cancer (update of QOL-BR23) [online] Available at: http://groups.eortc. be/qol/eortc-qol-module-breast-cancer-update-qol-br23 [Accessed 1 May 2018].

10. Madrid.org. (2019). Hospital La Paz. [online] Available at: http:// www.madrid.org/cs/Satellite?c=Page\&cid=1354193178794\&pa-

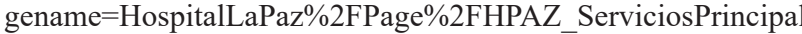
[Accessed 17 Nov. 2019].

11. Eltahir Y, Werners L, Dreise MM, et al. Which breast is the best? Successful autologous or alloplastic breast reconstruction: patient-reported quality-of-life outcomes. Plast. Reconst. Surg,. $2015 ; 135: 43-50$.
12. Yueh JH, Slavin SA, Adesiyun T, et al. Patient satisfaction in postmastectomy breast reconstruction: a comparative evaluation of DIEP, TRAM, latissimus flap and implant techniques. Plast Reconst. Surg. 2010;125(6):1585-1595.

13. Varela $\mathbf{R}$, Landin $\mathbf{L}$, Bonastre $\mathbf{J}$, Bolado $\mathbf{P}$, et al. Seguridad y complicaciones en reconstrucción mamaria autóloga con colgajo DIEP: una revisión sistemática. Rev Senol Patol Mamar. 2017; 30(3):119-129.

14. Gabriel SE, Woods JE, O'Fallon WM, et al. Complications leading to surgery after breast implantation. NEng J Med, 1997; 336(10): 677-682.

15. Handel N, Jensen JA, Black Q, et al. The fate of breast implants: a critical analysis of complications and outcomes. Plast. Reconst. Surg 1995;96 (7):1521-1533.

16. Reza Goyanes M, Andradas Aragonés E, Blasco Amaro JA Reconstrucción mamaria inmediata. Revisión sistemática y evaluación de resultados en una unidad de RMI de la Comunidad de Madrid. Madrid: Unidad de Evaluación de Tecnologías Sanitarias (UETS), Agencia Laín Entralgo; noviembre 2005.

17. Sullivan SR, Fletcher D, Isom $C$, et al. True incidence of all complications following immediate and delayed breast reconstruction. Plast. Reconst. Surg 2008;122(1): 19-28.

18. Alderman A, Wilkins E, Hyungjin MK, et al. Complications in postmastectomy breast reconstruction: two-year results of the Michigan Breast Reconstruction Outcome Study. Plast. Reconst. Surg 2002;(7): 2265-2274.

19. Jajsi R, Jiang J, Momoh AO, Alderma A, et al. Complications after mastectomy and inmediate breast reconstruction for breast cancer: a claims-based analysis. Ann Surg. 2016;263:219-227.

20. McCarthy CM, Mehrara BJ, Riedel E, Davidge K, et al. Predicting complications following expander/implant breast reconstruction: an outcomes analysis based on preoperative clinical risk. Plast. Reconst. Surg, 2008:121(6):1886-1892.

21. Renovación y reparación tisular: regeneración, curación y fibrosis. Patología estructural y funcional. $7^{\text {a }}$ Edición. Barcelona: Elsevier. Pp. 107-113.

22. EORTC - Quality of Life (2018). Breast Reconstruction [online] Available at: http://qol.eortc.org/questionnaire/qlq-brecon23/ [Accessed 19 May 2018]. 